\title{
Scaling behavior of optimally structured catalytic microfluidic reactors
}

\author{
Okkels, Fridolin; Bruus, Henrik
}

Published in:

Physical Review E

Link to article, DOI:

10.1103/PhysRevE.75.016301

Publication date:

2007

Document Version

Publisher's PDF, also known as Version of record

Link back to DTU Orbit

Citation (APA):

Okkels, F., \& Bruus, H. (2007). Scaling behavior of optimally structured catalytic microfluidic reactors. Physical Review E, 75(1), 016301. https://doi.org/10.1103/PhysRevE.75.016301

\section{General rights}

Copyright and moral rights for the publications made accessible in the public portal are retained by the authors and/or other copyright owners and it is a condition of accessing publications that users recognise and abide by the legal requirements associated with these rights.

- Users may download and print one copy of any publication from the public portal for the purpose of private study or research.

- You may not further distribute the material or use it for any profit-making activity or commercial gain

- You may freely distribute the URL identifying the publication in the public portal

If you believe that this document breaches copyright please contact us providing details, and we will remove access to the work immediately and investigate your claim. 


\title{
Scaling behavior of optimally structured catalytic microfluidic reactors
}

\author{
Fridolin Okkels and Henrik Bruus \\ MIC-Department of Micro and Nanotechnology, NanoDTU, Technical University of Denmark, Building 345 East, \\ DK-2800 Lyngby, Denmark \\ (Received 30 June 2006; published 8 January 2007)
}

\begin{abstract}
In this study of catalytic microfluidic reactors we show that, when optimally structured, these reactors share underlying scaling properties. The scaling is predicted theoretically and verified numerically. Furthermore, we show how to increase the reaction rate significantly by distributing the active porous material within the reactor using a high-level implementation of topology optimization.
\end{abstract}

DOI: 10.1103/PhysRevE.75.016301 PACS number(s): 47.70.Fw, 02.60.Pn, 47.61.-k, 82.33.Ln

Chemical processes play a key role for the production and analysis of many substances and materials needed in industry and health care. Generally, the optimization of these processes is an important goal, and with the introduction of microfluidic reactors involving laminar flows, the resulting concentration distributions mean better control and utilization of the reactors [1]. These conditions make it possible to design reactors using the method of topology optimization [2], which recently has been applied to fluidic design of increasing complexity [3-5].

First, we report the finding of scaling properties of such optimal reactors. To illustrate the method we study a simple model of a chemical reactor, in which the desired product arises from a single first-order catalytic reaction due to a catalyst immobilized on a porous medium filling large regions of the reactor.

Next, we show that topology optimization can be employed to design optimal chemical microreactors. The goal of the optimization is to maximize the mean reaction rate of the microreactor by finding the optimal porosity distribution of the porous catalytic support. Despite the simplicity of the model, our work shows that topology optimization of the design of the porous support inside the reactor can increase the reaction rate significantly.

Our model system is a first-order catalytic reaction, $A \stackrel{C}{\rightarrow} B$, taking place inside a microfluidic reactor of length $L$, containing a porous medium of spatially varying porosity $\gamma(\mathbf{r})$ and a buffer fluid filling the pores. The porosity $\gamma$ is defined as the local volume fraction occupied by the buffer fluid [6], and it can vary continuously from zero to unity, where $\gamma=0$ is the limit of dense material (vanishingly small pores) and $\gamma=1$ is the limit of pure fluid (no porous material). The reactant $A$ and the product $B$ exist only in dissolved form with concentrations $a$ and $b$, respectively, in the buffer fluid, which is driven through the reactor by a constant, externally applied pressure difference $\Delta p$ between an inlet and outlet channel. The catalyst $C$ is immobilized with concentration $c$ on the porous support.

The working principle of the reactor is quite simple. The buffer fluid feeds pure reactant $A$ to the porous medium supporting the catalyst $C$. The reaction rate is high if at the same time the reactant $A$ is supplied at a high rate and the amount of immobilized catalyst $C$ is large. However, these two conditions are contradictory. For a given pressure drop $\Delta p$ the supply rate of $A$ is high if $\gamma$ is high, allowing for a large flow rate of the buffer fluid. Conversely, the amount of catalyst $C$ is high if $\gamma$ is low, corresponding to a dense porous support with a large active region. Consequently, an optimal design of the porous support must exist involving intermediate values of the porosity. Besides, the optimal design may involve an intricate distribution of porous support within the reactor, and to find this we employ the method of topology optimization in the implementation of Ref. [5].

In the steady-state limit, the reaction kinetics is given by the following advection-diffusion-reaction equation for the reactant concentration $a$,

$$
[\mathbf{u}(\gamma) \cdot \boldsymbol{\nabla}] a=D \nabla^{2} a-k(\gamma) a .
$$

Here $\mathbf{u}(\gamma)$ is the velocity field of the buffer fluid, $D$ is the diffusion constant of the reactant in the buffer, and $-k(\gamma) a$ is the reaction term of the first order isothermal reaction, which depends on the concentration of the catalyst $C$ through $\gamma(\mathbf{r})$. In this problem three characteristic time scales $\tau_{A}, \tau_{R}$, and $\tau_{D}$ naturally arise,

$$
\tau_{A}=\frac{L}{u}, \quad \tau_{D}=\frac{L^{2}}{D}, \quad \tau_{R}=\frac{1}{k_{\Omega}},
$$

which correspond directly to the advection, diffusion, and reaction term in Eq. (1), respectively. These time scales will be used in the following theoretical analysis. Note that the index of $\Omega$ generally denotes an average over the design region, e.g., $k_{\Omega}=\langle k(\gamma)\rangle_{\Omega}$.

The porosity field $\gamma(\mathbf{r})$ uniquely characterizes the reactor design since it determines both the distribution of the catalyst and the flow of the buffer. In the Navier-Stokes equation, governing the flow of the buffer, the presence of the porous support can be modeled by a Darcy damping force density $-\alpha(\gamma) \mathbf{u}$, where $\alpha$ is the local, porosity-dependent, inverse permeability [7]. Assuming further that the buffer fluid is an incompressible liquid of density $\rho$ and dynamic viscosity $\eta$, the governing equations of the buffer in steady-state become

$$
\begin{gathered}
\rho(\mathbf{u} \cdot \nabla) \mathbf{u}=-\nabla p+\eta \nabla^{2} \mathbf{u}-\alpha(\gamma) \mathbf{u}, \\
\boldsymbol{\nabla} \cdot \mathbf{u}=0 .
\end{gathered}
$$

The coupling between $\alpha$ and $\gamma$ is given by the function $\alpha(\gamma) \equiv \alpha_{\max } \frac{q(1-\gamma)}{q+\gamma}$, where $\alpha_{\max }$ is determined by the nondimensional Darcy number $D a=\frac{\eta}{\alpha_{\max } L^{2}}$, and $q$ is a positive 
parameter used to ensure global convergence of the topology optimization method $[3,5]$. In this work $D a$ is typically around $10^{-5}$, resulting in a strong damping of the buffer flow inside the porous support. The model is solved for a given $\gamma(\mathbf{r})$ by first finding $\mathbf{u}(\gamma)$ from Eqs. (3a) and (3b) and then $a(\mathbf{r})$ from Eq. (1).

Our aim is to optimize the average reaction-rate $(k(\gamma) a)_{\Omega}$ of the reactor by finding the optimal porosity field $\gamma(\mathbf{r})$. We therefore introduce the following objective function $\Phi(\gamma)$, which by convention has to be minimized,

$$
\Phi(\gamma)=-(k(\gamma) a)_{\Omega} .
$$

To better characterize the performance of the reactor and to introduce the related quantities, we first analyze a simple one-dimensional (1D) model defined on the $x$ axis. The porous medium is placed in the reaction region $\Omega$ extending from $x=0$ to $x=L$. Equation (3b) leads to a constant flow velocity $u$, and as the complete pressure drop occurs in the porous medium, we have $p(0)=p_{0}+\Delta p$ and $p(L)=p_{0}$. In this case the boundary conditions for the advectiondiffusion-reaction equation (1) are $a(-\infty)=a_{0}, a^{\prime}(-\infty)=0$, and $a^{\prime}(\infty)=0$, where the primes indicate $x$ derivatives. We denote the outlet concentration $a(\infty)=a_{L}$. From Eqs. (1) and (4), we then derive the following expression of the objective function:

$$
\begin{aligned}
\Phi(\gamma) & =-\langle k(\gamma) a\rangle=\frac{1}{L} \int_{0}^{L}\left[u(\gamma) a^{\prime}-D a^{\prime \prime}\right] d x \\
& =\frac{u(\gamma)}{L}\left(a_{L}-a_{0}\right)-\frac{D}{L}\left[a^{\prime}(L)-a^{\prime}(0)\right] .
\end{aligned}
$$

For simplicity, we now limit the analysis to the nondiffusive case $(D=0)$, and from Eq. (5) we obtain the objective function defined in terns of the reaction conversion $\mathcal{C}$,

$$
\Phi(\gamma)=-\frac{u(\gamma) a_{0}}{L} \mathcal{C}, \quad \text { with } \mathcal{C} \equiv 1-\frac{a_{L}}{a_{0}} .
$$

With an explicit $x$ dependence of the reaction rate coefficient $k(x)$, we obtain $u(\gamma) a^{\prime}=-k(x) a$ with the solution

$$
a(x)=a_{0} e^{-K(x) / u(\gamma)}, \quad \text { with } K(x) \equiv \int_{0}^{x} k(\widetilde{x}) d \widetilde{x} .
$$

This leads to the following expression of the conversion:

$$
\mathcal{C}=1-e^{-K(L) / u(\gamma)}=1-e^{-\left[k_{\Omega} / u(\gamma)\right] L}=1-e^{-\delta},
$$

where we have introduced the dimensionless Damköhler number $\delta[8]$

$$
\delta \equiv \frac{\tau_{A}}{\tau_{R}}=\frac{k_{\Omega}}{u(\gamma)} L,
$$

having the physical interpretation of the ratio between the advection and the reaction time scale.

To derive the flow speed $u(\gamma)$ in the 1D model we first let $q \rightarrow \infty$, resulting in $\alpha(\gamma)=\alpha_{\max }(1-\gamma)$, and then by integrating Eq. (3a), $0=-p^{\prime}-\alpha_{\max }(1-\gamma) u$, we obtain

$$
u=\frac{\Delta p}{\alpha_{\max }\left(1-\gamma_{\Omega}\right) L}=\frac{D a}{1-\gamma_{\Omega}} \frac{\Delta p L}{\eta} .
$$

To solve the 1D optimization problem analytically, we chose to abandon the spatial variations of $\gamma$ in the 1D model. We have to find the solutions to $\frac{\partial \Phi}{\partial \gamma_{\Omega}} \equiv 0$, and from Eqs. (6) and (8), we end up by having to solve the following equation:

$$
1-e^{\delta}+\delta(1+\beta)=0,
$$

where we have assumed that $k\left(\gamma_{\Omega}\right) \propto\left(1-\gamma_{\Omega}\right)^{\beta}$. The specific properties of the catalytic reaction determines the value of $\beta$, e.g., if the full volume of the porous medium is active then $\beta=1$, while if only the surface is active then $\beta=2 / 3$. Solving Eq. (11) gives the optimal value of $\delta_{\beta}$, where the reference to $\beta$ now is explicit.

All numerical solutions are found using the commercial numerical modeling tools MATLAB [9] and COMSOL [10]. To validate numerically the analytic results of the $1 \mathrm{D}$ model, we solve Eqs. (1) and (10) for a given homogeneous design variable $\gamma_{\Omega}$ and find the optimal value using a brute-force optimization method [11].

To obtain a general scaling parameter for the problem defined in Eq. (1) we reintroduce diffusion. However, to minimize the trivial influence from the inlet and outlet, we only study the limit of low diffusion, e.g., $\tau_{D} \gg \tau_{A}, \tau_{R}$. In this limit the optimal reactors involve a balance between the advection and reaction processes, and consequently we expect that $\tau_{A}$ and $\tau_{R}$ should enter on equal footing in the scaling parameter. We are therefore led to propose the following dimensionless form of the scaling parameter:

$$
\frac{\sqrt{\tau_{A} \tau_{R}}}{\tau_{D}}=\frac{D}{\sqrt{k\left(\gamma_{\Omega}\right) u\left(\gamma_{\Omega}\right) L^{3}}} .
$$

Figure 1 shows that the measured values of $\delta_{\beta}$ and $\mathcal{C}_{\beta}$ for optimal porosity both scale with respect to $\sqrt{\tau_{A} \tau_{R}} / \tau_{D}$. The simulations cover 512 optimal reactors in a wide and dense parameter scan [12], and as they collapse almost perfectly on single curves, we have not distinguished the data points further. In the nondiffusive case $D=0$ and $\sqrt{\tau_{A} \tau_{R}} / \tau_{D}=0$, exact values of $\delta_{\beta}$ and $\mathcal{C}_{\beta}$ are determined by Eqs. (8) and (11), and they match exactly with the numerical results, as seen in Fig. 1 , where they are marked by circles on the ordinate.

We now introduce three types of $2 \mathrm{D}$ reactors: the uniform reactors, Fig. 2(a), the membrane reactors, Fig. 2(b), and the topology optimized reactors, for which a few are shown in Fig. 3. First we optimize the simple reactors in Fig. 2. They both depend only on one variable, which for the uniform reactor is the uniform porosity $\tilde{\gamma}$, and for the membrane reactor is the width $\ell$ of a porous membrane of porosity $\gamma^{*} \equiv 0$ [11]. Because of mirror symmetry in the $x z$ plane, only the upper half of the reactors are solved in all the following work.

In the third type of 2D reactors we let the porosity $\gamma(\mathbf{r})$ vary freely within the same design region as for the uniform reactor. The optimal design is found using the topology optimization method, described in detail in Ref. [5]. This is an iterative method, in which, starting from an initial guess $\gamma_{0}$ 


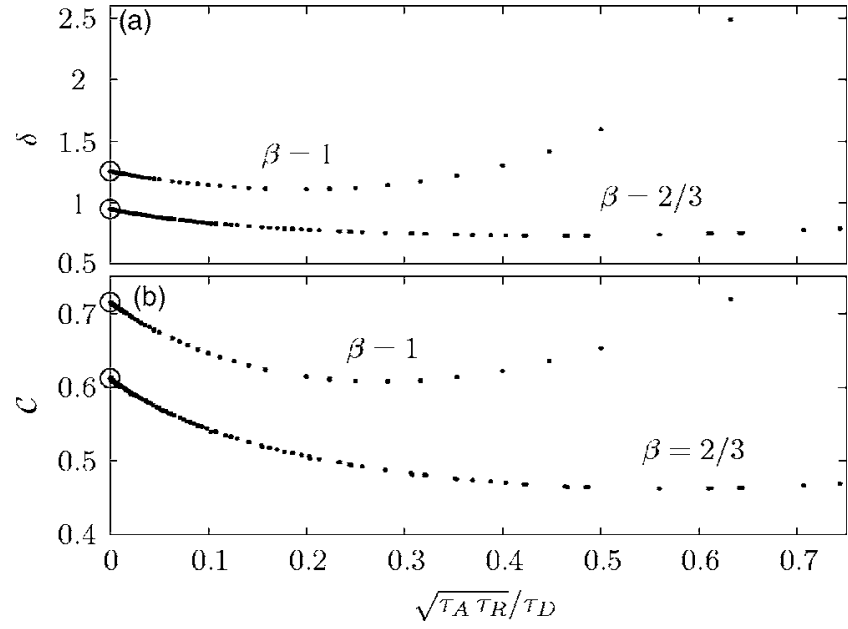

FIG. 1. Plot of 1D results showing (a) Damköhler number $\delta$ and (b) conversion $\mathcal{C}$, both as a function of $\sqrt{\tau_{A} \tau_{R}} / \tau_{D}$ and for optimal choices of porosity. In both cases $\beta=2 / 3,1$ and the parameter scan of each choice consists of 512 optimizations [12], which collapse nicely. For zero difffusion, $\sqrt{\tau_{A} \tau_{R}} / \tau_{D}=0$, Eqs. (11) and (8) give the exact results $\delta_{2 / 3}=0.9474, \quad \delta_{1}=1.2564, \quad \mathcal{C}_{2 / 3}=0.6122$, and $\mathcal{C}_{1}=0.7153$, which are marked by circles on the ordinate axis.

of the design variable, the $n$th iteration consists of first solving the systems for the given design variable $\gamma_{n}$, then evaluating the sensitivities $\frac{\partial \Phi}{\partial \gamma}$ by solving a corresponding adjoint problem, and finally obtaining an improved updated $\gamma_{n+1}$ by use of the "method of moving asymptotes" (MMA) $[13,14]$. In Fig. 3 is shown a representative collection of topology optimized designs together with the corresponding flow speed $u$, concentration $a$, reaction rate $k(\gamma) a$, and parameter values. In the large parameter space under investigation, our work shows a systematic decrease of pore sizes and the emergence of finer structures in the topology optimized reactors as the scaling parameter $\sqrt{\tau_{A} \tau_{R}} / \tau_{D}$ is decreased.

In Fig. 4 the conversion $\mathcal{C}$ is plotted as a function of $\sqrt{\tau_{A} \tau_{R}} / \tau_{D}$ for all optimal reactors of this work. It shows that all reactors collapse on curves similar to the $1 \mathrm{D}$ reactors, although the topology optimized reactors exhibit a larger spread. We believe that this scaling is a signature of a general property of optimal immobilized catalytic reactors. Note that the conversion of the uniform reactor in the low diffusion limit is a few percent higher than the theoretical estimate, an effect caused by low convection in the corners, resulting in "dead zones."

In terms of the objective function $\Phi$, the topologically

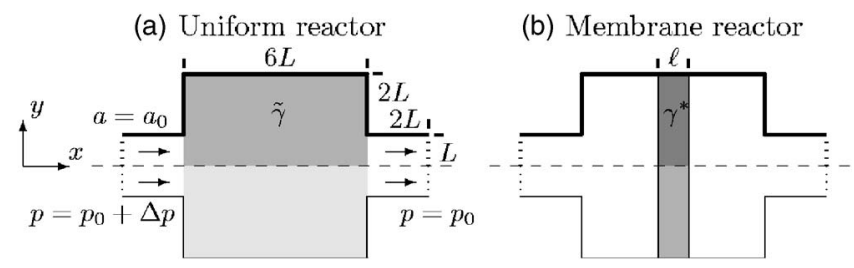

FIG. 2. Illustration of the two simple 2D reactor setups, (a) the uniform reactor with porosity $\tilde{\gamma}$ and (b) the membrane reactor of width $\ell$ and with porosity $\gamma^{*} \equiv 0$. The horizontal dashed line is a symmetry line.

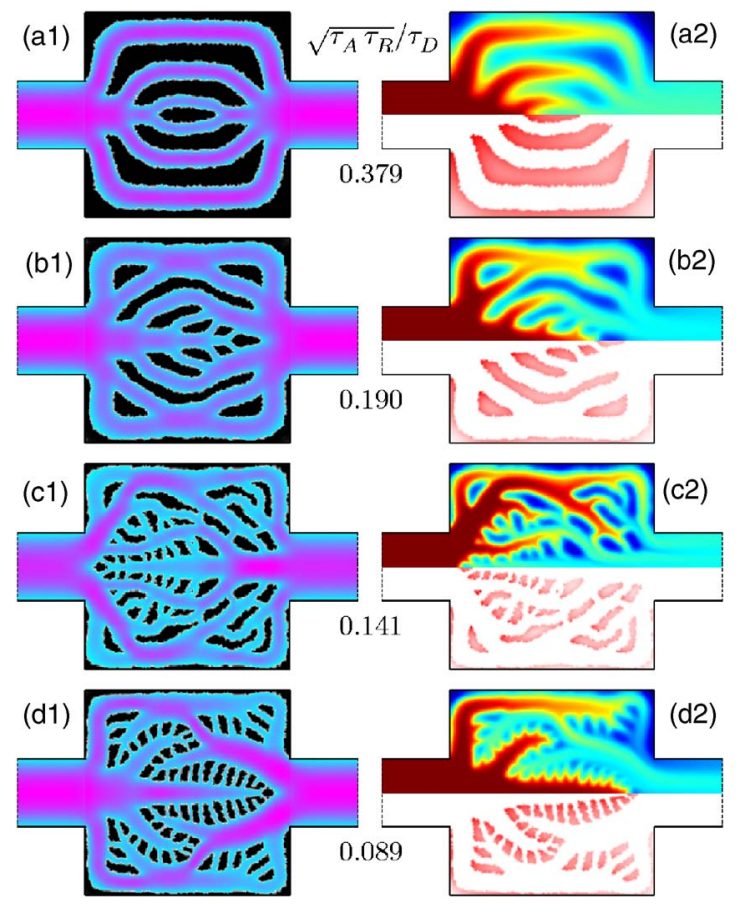

FIG. 3. (Color online) Representative collection of topology optimized reactor designs for deceasing values of $\sqrt{\tau_{A} \tau_{R}} / \tau_{D}$. (Left column) The distribution $\gamma$ of porous material in black together with a color-grading indication of the flow speed $u$. (Right column) The concentration $a$ on top with the reaction rate $k(\gamma) a$ below. Parameters (in SI units) $L=10^{-3}$ and the following values of $\left[D a, D, \Delta p, k_{a}\right]$ : (a) $\left[10^{-4}, 3 \times 10^{-8}, 0.25,0.25\right]$, (b) $\left[10^{-4}, 3 \times 10^{-8}, 0.25,1\right]$, (c) $\left[10^{-5}, 10^{-8}, 0.5,1\right]$, and (d) $\left[10^{-4}, 10^{-8}, 0.25,0.5\right]$.

optimized reactors are significantly improved compared to the simple $2 \mathrm{D}$ reactors. To investigate the nature behind this improvement, we show in Fig. 5 a log-log plot of the flow rate $Q$ and the conversion $\mathcal{C}$ normalized by the values $\mathcal{C}_{\text {unif }}$ and $Q_{\text {unif }}$ of the uniform reactors at the same parameters. Because Eq. (6) gives the following scaling of the objective function $\Phi \sim Q \mathcal{C}$, the rate of improvement with respect to the

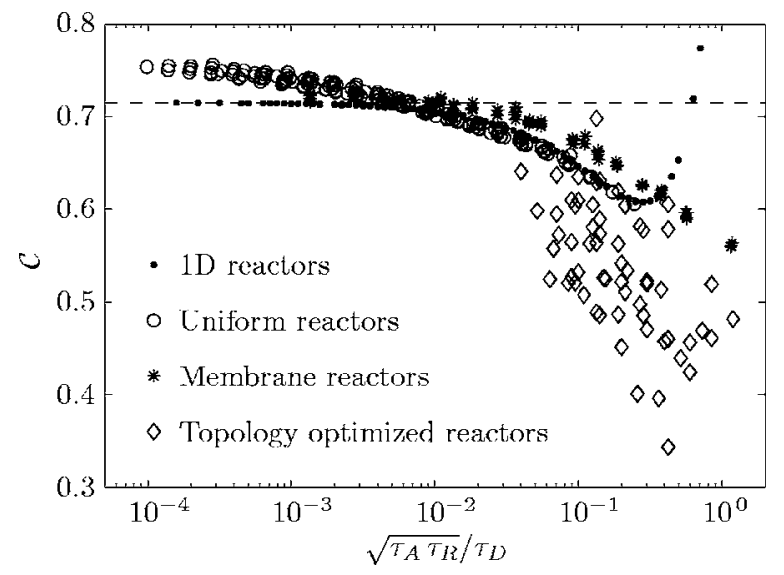

FIG. 4. Overall scaling of the conversion $\mathcal{C}$ as a function of $\sqrt{\tau_{A} \tau_{R}} / \tau_{D}$ for the different optimal reactors. The abscissa is logarithmic to emphasize the common scaling behavior. The dashed line indicates the theoretical value $\mathcal{C}_{1}$ for zero diffusion in the $1 \mathrm{D}$ case. 


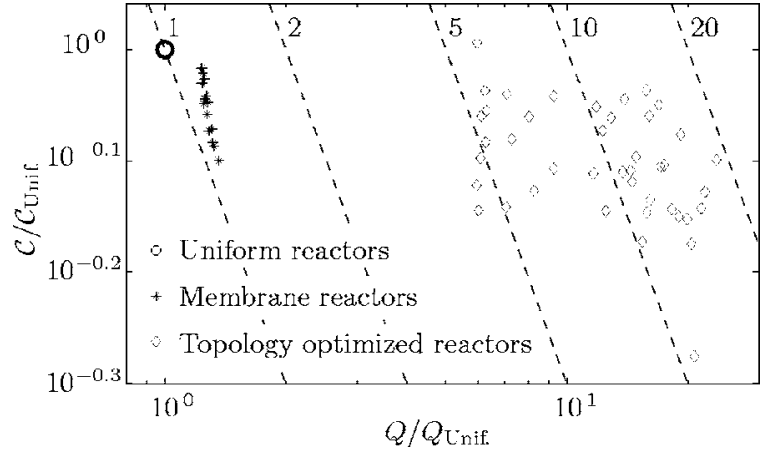

FIG. 5. Log-log plot of the relation between convection $\mathcal{C}$ and flow rate $Q$ for the different reactors, when normalized with values $\mathcal{C}_{\text {unif }}$ and $Q_{\text {unif }}$ of the uniform reactors. The reaction rate improvements are shown at the top (see text).

uniform reactors can be read off directly, as the contours of the improvement factors of $\Phi$ become straight lines, as shown by the dashed lines labeled by the corresponding factors in Fig. 5. It is seen that topology optimization can increase the reaction rate of the optimal reactors by nearly a factor of 20, and furthermore it does so by increasing the flow rate at the expense of lower conversions. The important insight thus gained is that the distribution of the advected reactant by the microfluidic channel network over a large area at minimal pressure loss plays a significant role when optimizing microreactors.

To conclude, we have analyzed a single first-order catalytic reaction in a laminar microfluidic reactor with optimal distribution of porous catalyst. The flow is pressure driven and the flow through the porous medium is modeled using a simple Darcy damping force. Our goal has solely been to optimize the average reaction rate, with no constraints on the conversion or the catalytic properties. A characterization of the optimal configuration has been derived theoretically and validated numerically. It shows a general scaling behavior, depending only on the reaction properties of the catalyst. The analysis is based on a very simple reaction since this emphasizes the point that the optimization of even simple reactions result in nontrivial scaling properties and complex optimal designs. Using topology optimization to design optimal reactors gives rise to reaction rate improvements of close to a factor of 20, compared to an corresponding optimal uniform reactor, and the improvement originates mainly due to an improved transport and distribution of the reactant. Furthermore, for the topology optimized reactors, we have found a systematic decrease of pore sizes and the emergence of finer structures as the scaling parameter is decreased. Our work points out a general and potentially very powerful method of improving microfluidic reactors.

F.O. was supported by Danish Technical Research Council Contracts No. 26-03-0037 and No. 26-03-0073.
[1] L. Kiwi-Minsker and A. Renken, Catal. Today 110, 2 (2005).

[2] M. P. Bendsøe and O. Sigmund, Topology OptimizationTheory, Methods and Applications (Springer, Berlin, 2003).

[3] T. Borrvall and J. Petersson, Int. J. Numer. Methods Fluids 41, 77 (2003).

[4] A. Gersborg-Hansen, O. Sigmund, and R. B. Haber, Struct. Multidiscip. Optim. 30, 181 (2005).

[5] L. H. Olesen, F. Okkels, and H. Bruus, Int. J. Numer. Methods Eng. 65, 975 (2006).

[6] G. Desmet, J. D. Greef, H. Verelst, and G. V. Baron, Chem. Eng. Sci. 58, 3187 (2003).

[7] J. Bear, Dynamics of Fluids in Porous Media (American Elsevier, New York, 1972).
[8] G. Damköhler, Chem.-Ing.-Tech. 3, 359 (1937).

[9] The MathWorks, Inc., www.mathworks.com

[10] COMSOL AB, www.comsol.com

[11] The MATLAB routine fminbnd minimizes a single variable using a golden section search and parabolic interpolation.

[12] Parameter scan: $\left[D, \Delta p, k_{a}\right]=\left[\chi_{D} D_{0}, \chi_{p} \Delta p_{0}, \chi_{k} k_{a 0}\right]$ with $\chi_{i}$ $=[0.1,0.2,0.5,1,2,5,10,20], i=D, p, k$, and $\left[D_{0}, \Delta p_{0}, k_{a 0}\right]$ $=\left[10^{-9}, 0.1,0.1\right], D a=10^{-4}$.

[13] K. Svanberg, Int. J. Numer. Methods Eng. 24, 359 (1987).

[14] A MATLAB implementation, mmasub, of the MMA optimization algorithm [13] can be obtained (free of charge for academic purposes) from Krister Svanberg, KTH, Sweden. E-mail address: krille@math.kth.se 